San Jose State University

SJSU ScholarWorks

Master's Projects

Master's Theses and Graduate Research

Spring 2018

\title{
Micro-expression Recognition using Spatiotemporal Texture Map and Motion Magnification
}

Shashank Shivaji Pawar

San Jose State University

Follow this and additional works at: https://scholarworks.sjsu.edu/etd_projects

Part of the Computer Sciences Commons

\section{Recommended Citation}

Pawar, Shashank Shivaji, "Micro-expression Recognition using Spatiotemporal Texture Map and Motion Magnification" (2018). Master's Projects. 610.

DOI: https://doi.org/10.31979/etd.bqc3-vy2c

https://scholarworks.sjsu.edu/etd_projects/610

This Master's Project is brought to you for free and open access by the Master's Theses and Graduate Research at SJSU ScholarWorks. It has been accepted for inclusion in Master's Projects by an authorized administrator of SJSU ScholarWorks. For more information, please contact scholarworks@sjsu.edu. 


\title{
Micro-expression Recognition using Spatiotemporal Texture
}

\author{
Map and Motion Magnification
}

\author{
A Writing Project \\ Presented to \\ The Faculty of the Department of Computer Science \\ San José State University \\ In Partial Fulfilment \\ of the Requirements for the Degree \\ Master of Computer Science \\ By \\ Shashank Shivaji Pawar \\ Spring 2018
}




\title{
(C)2018
}

\author{
Shashank Shivaji Pawar
}

\section{ALL RIGHTS RESERVED}




\section{SAN JOSÉ STATE UNIVERSITY}

The Undersigned Thesis Committee Approves the Thesis Titled

Micro-expression Recognition using Spatiotemporal Texture Map and Motion Magnification

By

Shashank Shivaji Pawar

APPROVED FOR THE DEPARMENT OF COMPUTER SCIENCE

Dr. Teng Moh, Department of Computer Science

$05 / 09 / 2017$

Dr. Thomas Austin, Department of Computer Science $\quad$ 05/09/2017

Mr. James Casaletto, Department of Computer Science 05/09/2017 


\begin{abstract}
Micro-expressions are short-lived, rapid facial expressions that are exhibited by individuals when they are in high stakes situations. Studying these micro-expressions is important as these cannot be modified by an individual and hence offer us a peek into what the individual is actually feeling and thinking as opposed to what he/she is trying to portray. The spotting and recognition of micro-expressions has applications in the fields of criminal investigation, psychotherapy, education etc. However due to micro-expressions' short-lived and rapid nature; spotting, recognizing and classifying them is a major challenge. In this paper, we design a hybrid approach for spotting and recognizing micro-expressions by utilizing motion magnification using Eulerian Video Magnification and Spatiotemporal Texture Map (STTM). The validation of this approach was done on the spontaneous micro-expression dataset, CASMEII in comparison with the baseline. This approach achieved an accuracy of $80 \%$ viz. an increase by $5 \%$ as compared to the existing baseline by utilizing 10-fold cross validation using Support Vector Machines (SVM) with a linear kernel.
\end{abstract}




\section{Acknowledgements}

I am extremely thankful to Dr. Teng Moh, who is instrumental in guiding me throughout the research period. Without his constant motivation and direction, this work would have never seen the light of day. I would also like to thank my friend and colleague, Kevin Iocca for his operational expertise and more importantly his pep-talks that kept me going. 


\section{Table of Contents}

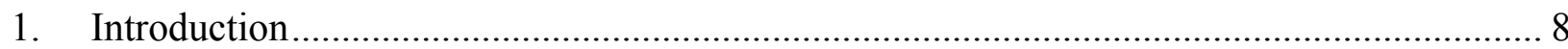

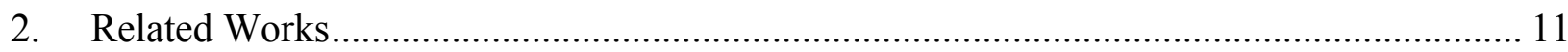

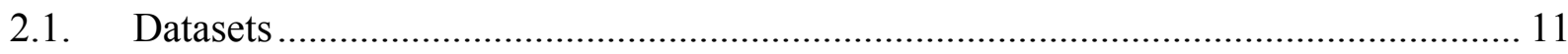

2.2. Preprocessing and Feature Extraction.................................................................... 13

2.3. Micro-expression Spotting \& Recognition ................................................................ 14

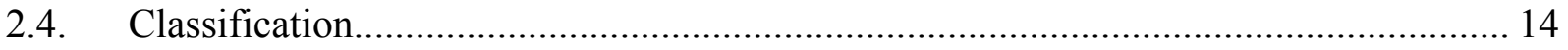

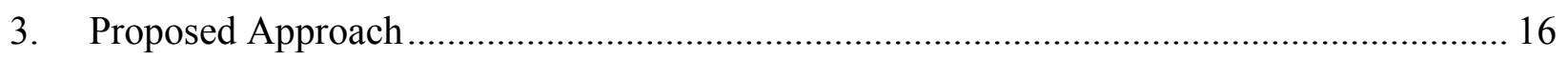

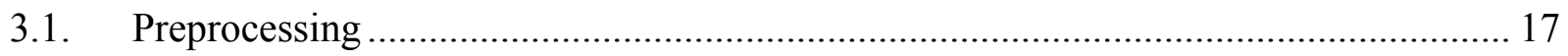

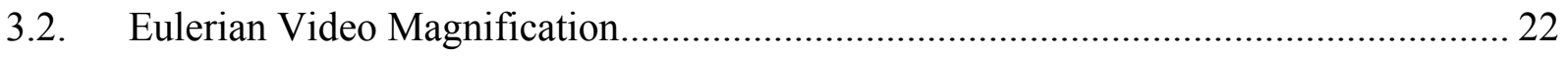

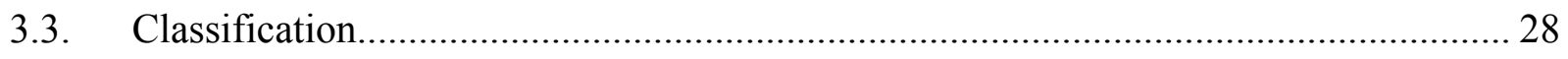

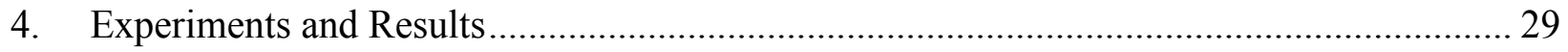

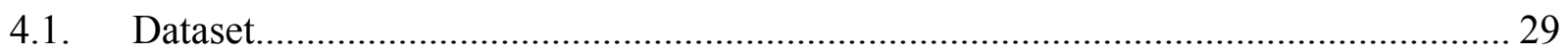

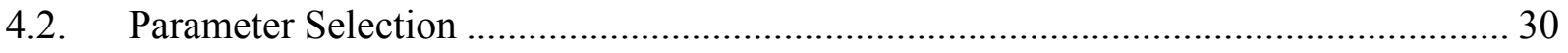

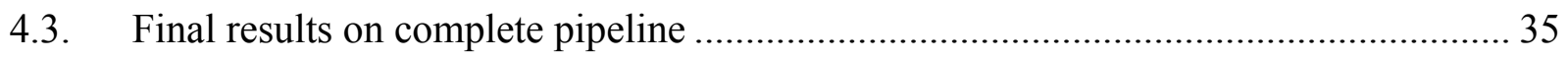

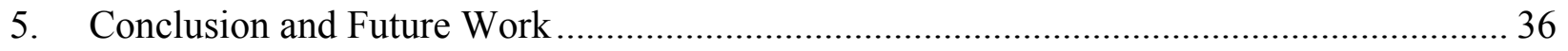

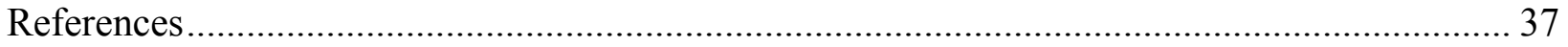




\section{Table of Figures}

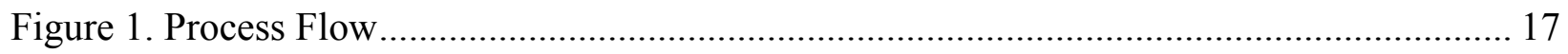

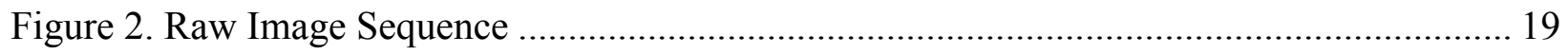

Figure 3. Preprocessed Image Sequence.................................................................. 20

Figure 4. Motion Magnified Image Sequence ........................................................................ 21

Figure 5. STTM Feature Extraction Process Flow ........................................................... 25

Figure 6. Process flow for block-based feature representation.......................................... 27

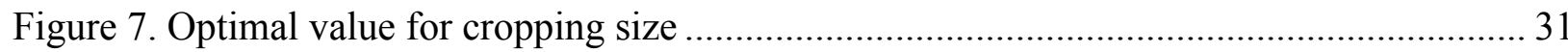

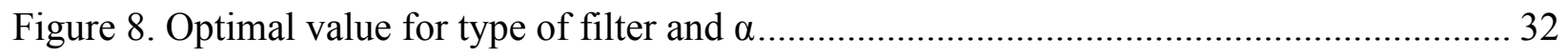

Figure 9. Optimal values of Kernel Shape and K-Fold validation ....................................... 34

\section{Table of Tables}

Table 1. Classification Report for 5 Emotion Categories .................................................... 35 


\section{Introduction}

Human beings are a social species. We have evolved to express our feelings and emotions through a variety of ways such as facial expressions, body language and hand gestures. Of all these, facial expressions are the most common way of expressing one's emotions [19]. Even while expressing verbally, research has shown that $55 \%$ of the total information expressed is with facial expressions [14]. These facts illustrate the importance of facial expressions in analyzing an individual's psyche and the inner workings of his/her mind.

Facial expressions are categorized as macro-expressions and micro-expressions. Macroexpressions are what an individual can control using their facial muscles, and hence they can be manipulated by the individual to suit his/her needs. They typically last longer and are easier to detect and recognize. Micro-expressions on the other hand, are involuntary and occur in highstakes situations [2]. They also last for much lesser durations, typically $1 / 25$ to $1 / 3$ second [13] [30]. What makes them worth studying is their ability to indicate the true thoughts and feelings of an individual, thus offering us a peek into his/her mind. In short, we can ascertain if their words corroborate with their true feelings. But due to the innate short duration of these micro-expressions, human beings find it extremely difficult to spot and recognize them [3]. A study has shown that even experts have a worse chance than a coin toss (about 40\%) of spotting a micro-expression [5].

Micro-expression spotting and recognition has applications in various fields right from criminal investigation, educational institutions to psychological evaluation. Law enforcement agencies could use micro-expression recognition techniques to ascertain if an individual is telling the truth instead of polygraph tests, which can be easily falsified [32] [33]. Individuals suffering from mental health issues such as autism [20] or schizophrenia [17] often cannot or do not want to express their emotions. Psychiatrists can use micro-expression techniques to diagnose the patient 
not only from his verbal cues, but from his non-verbal cues as well. In educational settings, this technique could be used by educators to gauge the class' understanding of his/her lectures.

While a lot of research has been done in the macro-expression recognition paradigm, microexpression recognition is still in its nascency. Spontaneous datasets such as SMIC [27] and CASMEII [29] are recent and important additions to this field. In addition to being one of the few spontaneous micro-expression datasets, CASMEII is also a video based dataset. This allows us to extract not only static but also dynamic information from the emotion sequence. While preparing the dataset, the authors of CASMEII used popular techniques such as Local Binary Pattern on Three Orthogonal Plane (LBP-TOP) [31] for feature extraction and Support Vector Machines (SVM) for classification. They achieved an accuracy of $63.41 \%$ for a 5 -class classification. Y. Wang et al [24] improved upon the work by W. Yan et al [29] by adding Eulerian Video Magnification (EVM) [25] as a preceding step to feature extraction using LBP-TOP. They achieved an accuracy of $75.30 \%$ using SVM classification with a Radial Basis Function kernel with leave-one-out-cross-validation (LOOCV).

While LBP-TOP is a popular method for feature extraction of dynamic textures, it has a few pitfalls. The feature descriptors created by LBP contain overlapping information due to overlapping of the features from the three orthogonal planes (XY, XT, YT). This results in a feature space that is computationally more expensive to classify [23].

In this paper, instead of using LBP-TOP as a method for feature extraction we use a 3D version of a Harris corner function [6] which makes use of textural spatiotemporal variations in video sequences called as a spatiotemporal texture map (STTM) [18]. Prior to extraction of the features using STTM, we also apply EVM [25] to magnify the subtleties in micro-expressions. We then use a SVM with a linear kernel using 10-fold cross validation for classification into five expression 
categories (Happiness, Surprise, Disgust, Repression and Others). Using this approach, we achieved an accuracy of $80 \%$.

This report is organized as follows: Section 2 discusses the research that has been done in the filed of macro and micro-expression spotting field. In Section 3, we discuss the proposed approach in detail. Section 4 enlists the experiments conducted and their results, to validate the hypotheses presented in Section 3. We conclude in Section 5, while pointing out certain directions in which this approach could be moved forward. 


\section{Related Works}

\subsection{Datasets}

A dataset that is a representative sample of the problem space is a prerequisite to implement an automated approach for solving problems using machine learning. Facial expression datasets are majorly divided into posed and spontaneous datasets. Unavailability of spontaneous datasets has been one of the major roadblocks in micro-expression analysis. While several posed datasets such as JAFFE [11], CK [7], MMI [16] exist for facial expression analysis, spontaneous microexpressions datasets are relatively rare. The primary difference between posed and spontaneous datasets is the mechanism in which they are elicited in test subjects. The main issue arises from the inherent nature of the micro-expressions. They are elicited in an individual only in high stakes situations. Ekman [2] suggests three ways to elicit micro-expressions in test subjects: (1) Asking the test subjects to lie about their reactions (2) Fabricating crime scenarios and (3) Asking the test subjects to lie about what they saw in videos. Despite these guidelines, a dataset that achieves micro-expressions elicitation is a recent addition to the field.

The literature shows a progressive trend in moving towards a dataset that captures microexpressions in the true sense of the word. In [26], Wu et al. use the Cohn-Kanade (CK) dataset. This dataset consists of 374 sequences of 97 subjects posing micro-expressions using the guidelines given above. However, on analyzing the dataset, it is evident that the image sequences are very aggressive and lose the inherent subtlety of a micro-expressions. In [28] and [24] Li et al. and Y. Wang et al respectively, have used a dataset called CASMEII [29]. This dataset is by far the most accurate in capturing the true essence of micro-expressions as it doesn't additionally instruct the test subjects. It lets the test subjects react naturally to the incident videos and captures their micro-expressions. This dataset contains 247 videos captured at $200 \mathrm{fps}$. 
In [18], Kamarol et al. employ a combined approach which uses multiple datasets to compare and contrast the results. In addition to $\mathrm{CK}+[10]$, an incremental update to the $\mathrm{CK}$ [7] dataset, it also uses the CASMEII dataset. The CK+ dataset contains a better sample of capturing the sample space than the CK dataset. It encompasses videos of test subjects from different ethnicities in a varied age group ranging from 18 to 50 . The $\mathrm{CK}+$ dataset consists of 304 labeled videos containing a total of 5521 frames. An interesting but a peculiar aspect of this paper is using the AFEW [1] dataset, which contains posed and spontaneous video clips from movies. This dataset contains about 601 videos containing 28,287 frames.

The literature describes the various thought processes and approaches employed to capture micro-expression datasets [7] [10] [29]. An upward trend in terms of image quality and frame rate is observed in micro-expressions dataset capturing techniques. Advancement in technology has also contributed to the increased quality in dataset preparation, as is evident from the $200 \mathrm{fps}$ capture of CASMEII dataset [29]. Increase in the frames-per-second characteristic of the videos is a step in the right direction as it will enable us to capture more information in the fleeting seconds and better capture micro-expressions. We also see a clear trend in improving the spontaneity of the datasets [7] [10] [29]. The initial datasets [7] [10] were posed and hence were not very descriptive of the micro-expression paradigm. However, the later datasets [29] have moved to a more spontaneous and natural expression elicitation techniques. Although some work has been done in trying to capture the various ethnicities' micro-expressions in the CK+ datasets [10], it still lacks in the spontaneity department. CASMEII dataset [29] scores in spontaneity but lacks the differences that can occur in varied age-groups and ethnicities. 


\subsection{Preprocessing and Feature Extraction}

Preprocessing is an important step in any form of image classification problem, as it levels the input space and makes the dataset as uniform as possible by removing unnecessary differences in illumination and other aspects. This enables us to analyze images for what they are without getting affected by local differences in the image quality. Once the images have been preprocessed, we need a mechanism to extract useful information from the images that can be quantified, convoluted and compared. The literature describes various approaches to extract these features.

Wu et al. [26] employ grey-scale conversion and detecting and cropping the face as preprocessing steps. The preprocessed images are $48 \times 48$ pixels in size. Wu et al. [26] use the algorithm described in [8] by Kienzle et al. to detect faces. Wu et al. [26] then utilize Gabor filters to extract features from the preprocessed images.

In contrast, Li et al. [28] do not employ any preprocessing to their images, as their technique for feature extraction thrives on a rich variety of the input image sequence. It employs the KanadeLucas-Tomasi algorithm [21] to detect faces. It then divides the image in $6 \times 6$ grid to extract its feature using Local Binary Pattern (LBP) [15] and Histogram of Optical Flow [9] techniques.

In [18] Kamarol et al. use an approach that is different from previous approaches of feature extraction, as it also utilizes the dynamic information of the video sequences that is present in the time dimension. Kamarol et al. [18] first crop the facial area in the image sequences using the Viola-Jones Face detection algorithm [22]. The features are then extracted using a Gaussian kernel and a Gaussian weighting function with second-moment matrices of the video sequences. 


\subsection{Micro-expression Spotting \& Recognition}

Pinpointing where in an image sequence the micro-expression occurs is called spotting. Once the micro-expression has been spotted, it has to be recognized to be belonging to one of the predefined categories in a process known as the micro-expression recognition.

The literature differs on how it spots and recognizes micro-expressions. The various algorithms that are used for feature extraction have their own strengths and weaknesses in addition to the richness of the feature set that they can utilize from the input dataset. LBP [15] is a popular method for feature extraction from images. G. Zhao and M. Pietikainen in [31] also extended it to the 3D space to include the dynamic component in their ME spotting and recognition approach. Gabor wavelets proposed by Lyons et al. [12] is another popular approach for textural representation.

\subsection{Classification}

Classifying the feature vectors extracted in the previous stages of these algorithms is the next logical step. One common aspect that arises from the literature in this scenario is regarding classification. Almost the entire literature utilizes Support Vector Machines (SVM) to classify the features albeit with a few minor differences.

Wu et al. [26] use the GentleBoost algorithm as a feature selector before using Linear SVM in an improved approach called as GentleSVM in 10-fold cross validation. Li. et al. [28] also utilize Linear SVM but with 5-fold cross validation. Kamarol et al. [18] utilize a multi-class SVM using one-against-one method. They use 2-fold cross validation and then employ Monte-Carlo simulation, repeating the process 100 times, till an average is achieved. Wang et al. [24] also use SVM with a RBF kernel using the LOOCV approach for classification 
As can be clearly observed from the literature, support vector machines are perfect for this type of problem statement as they provide good test measures with reasonable flexibility. 


\section{Proposed Approach}

In this paper, we propose a hybrid approach using the work done by Wang et al. [24], Wu et al. [25] and Kamarol et al. [18]. We use the Eulerian Video Magnification method described by Wu et al. [25] as a preprocessing step to amplify the micro-expressions as done by Wang et al. [24]. We then provide these motion magnified images to the algorithm defined by Kamarol et al. in [18] for feature extraction. We then use SVM with 10-fold cross validation with a linear kernel to classify the features into five expression categories.

The following steps briefly describe the methodology that will be employed in this project to spot, recognize and categorize micro-expressions:

1) Capture live video feed of test subjects being interviewed. The questions asked in the interview will be such that they will elicit micro-expressions in the test subjects

2) Crop input video using Viola-Jones facial detection method

3) Motion magnification of input video using Butterworth filter

4) The amount of magnification to be introduced is determined by a tunable parameter $\alpha$. We arrive at an empirically derived value of $\alpha$ which maximizes accuracy

5) Compute linear scale representation $(L)$ of input video using Gaussian kernel

6) Compute second moment matrix of $L$

7) Compute $\mu$ using the second-moment matrices and a Gaussian weighting function

8) Compute the $\operatorname{det}(\mu)$ and trace $(\mu)$

9) Compute the STTM

10) The features are then classified using a multi-class Support Vector Machine model with a linear kernel and 10-fold cross validation

Figure. 1 summarizes the process flow of the proposed approach. 


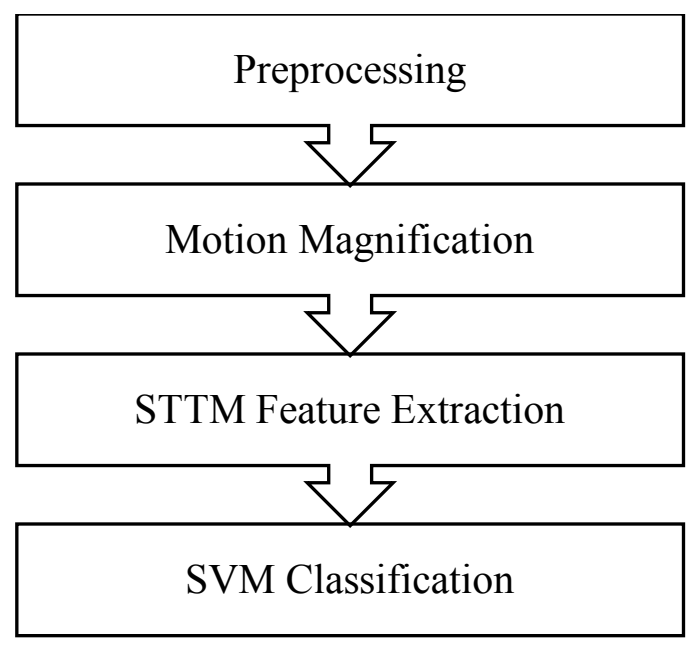

Figure 1. Process Flow

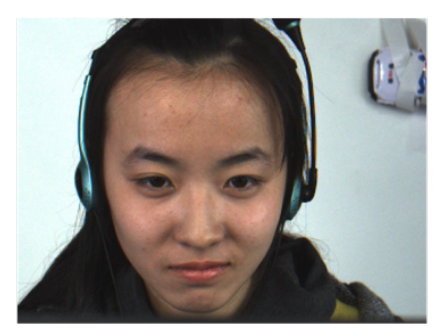

Raw Image

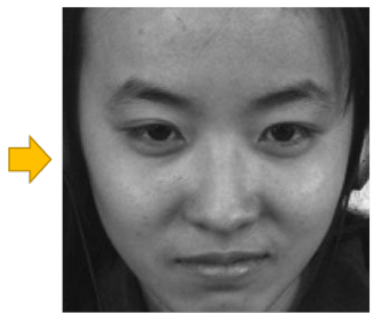

Preprocessed Image

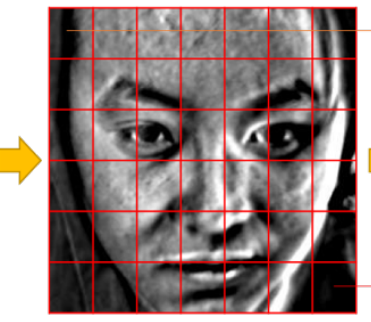

Motion Magnified Image

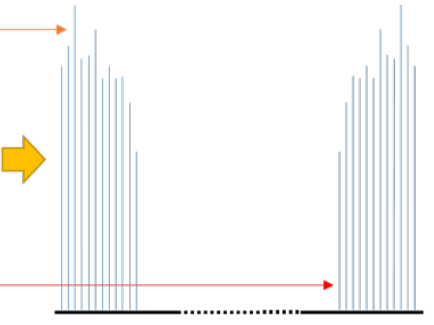

Feature Representation

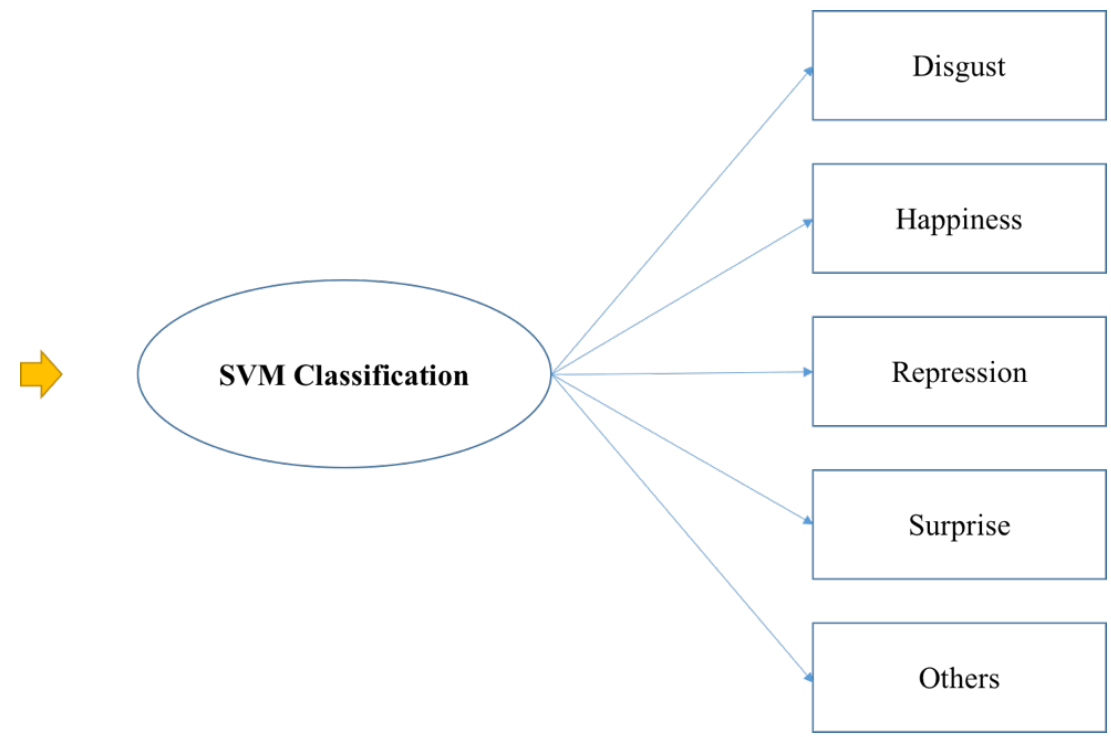

Figure 2. Visual Representation of the Proposed Approach 


\subsection{Preprocessing}

In order to improve the chances of micro-expression recognition, it is imperative to remove parts of the image sequence or videos that do not contribute to the end goal. Features such as hair, the color of the subjects' clothing, the subjects' background are all potential causes for introduction of noise to the feature space. Hence, as a first step, we remove these from the image sequences.

We detect the face of the subject using the Viola-Jones face detection algorithm [22]. In the CASMEII dataset, the position of the face is fairly constant and does not move much. Hence there is no need for facial alignment. After detecting the face, we crop the image and resize it an empirically derived resolution of $275 \times 275$. As we will see in Section 4, the resizing of the image to a particular resolution also affects the overall accuracy of the model.

After we have cropped the face, we then convert the image from RGB to grayscale. Information in the RGB range does not affect the overall accuracy of the model, but significantly reduces the computational complexity for feature extraction. Besides, as the feature extraction makes use of the textural information in the image sequences, keeping the image sequence in RGB color range is inconsequential. 

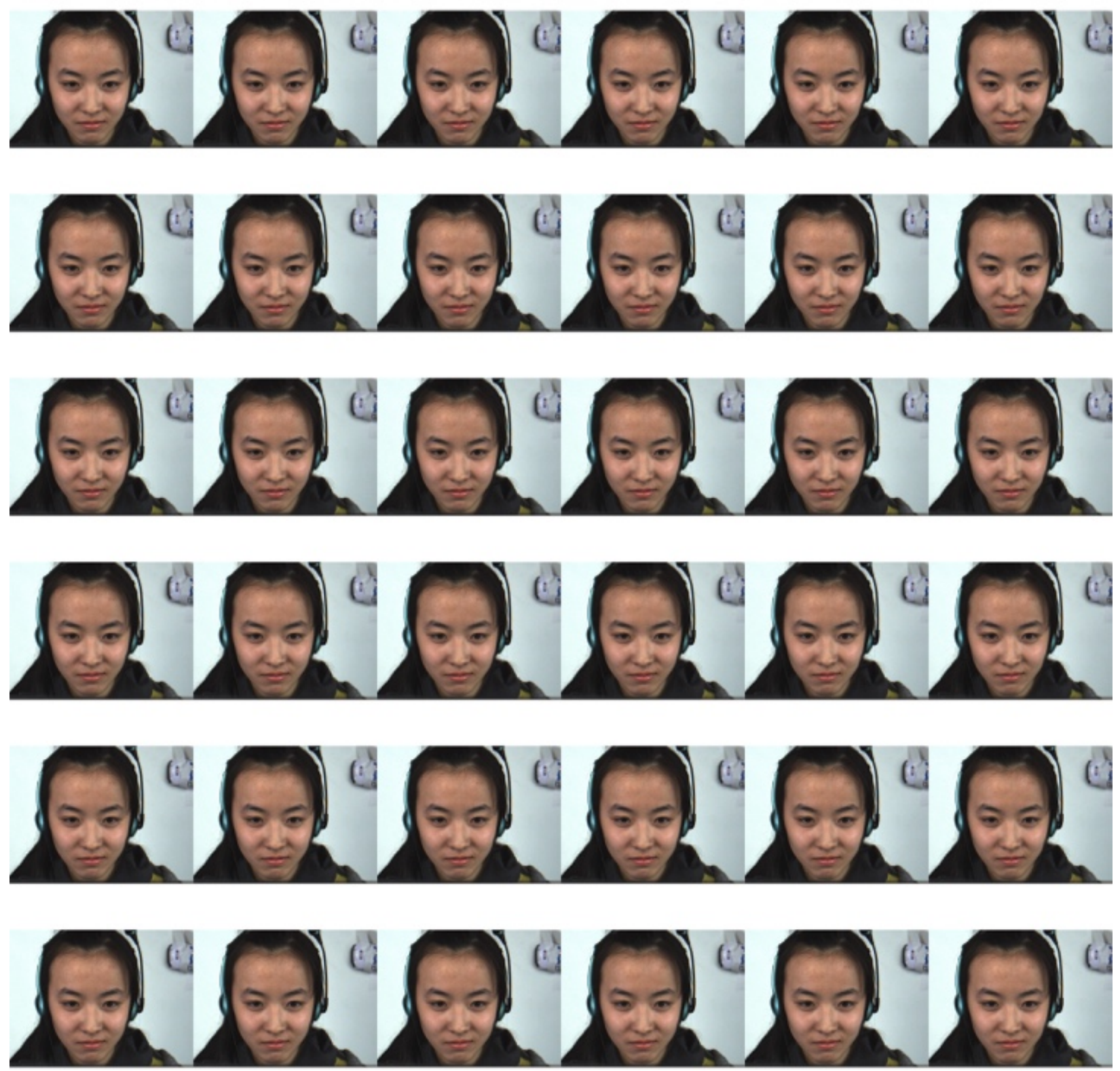

Figure 3. Raw Image Sequence 
CS298 Report

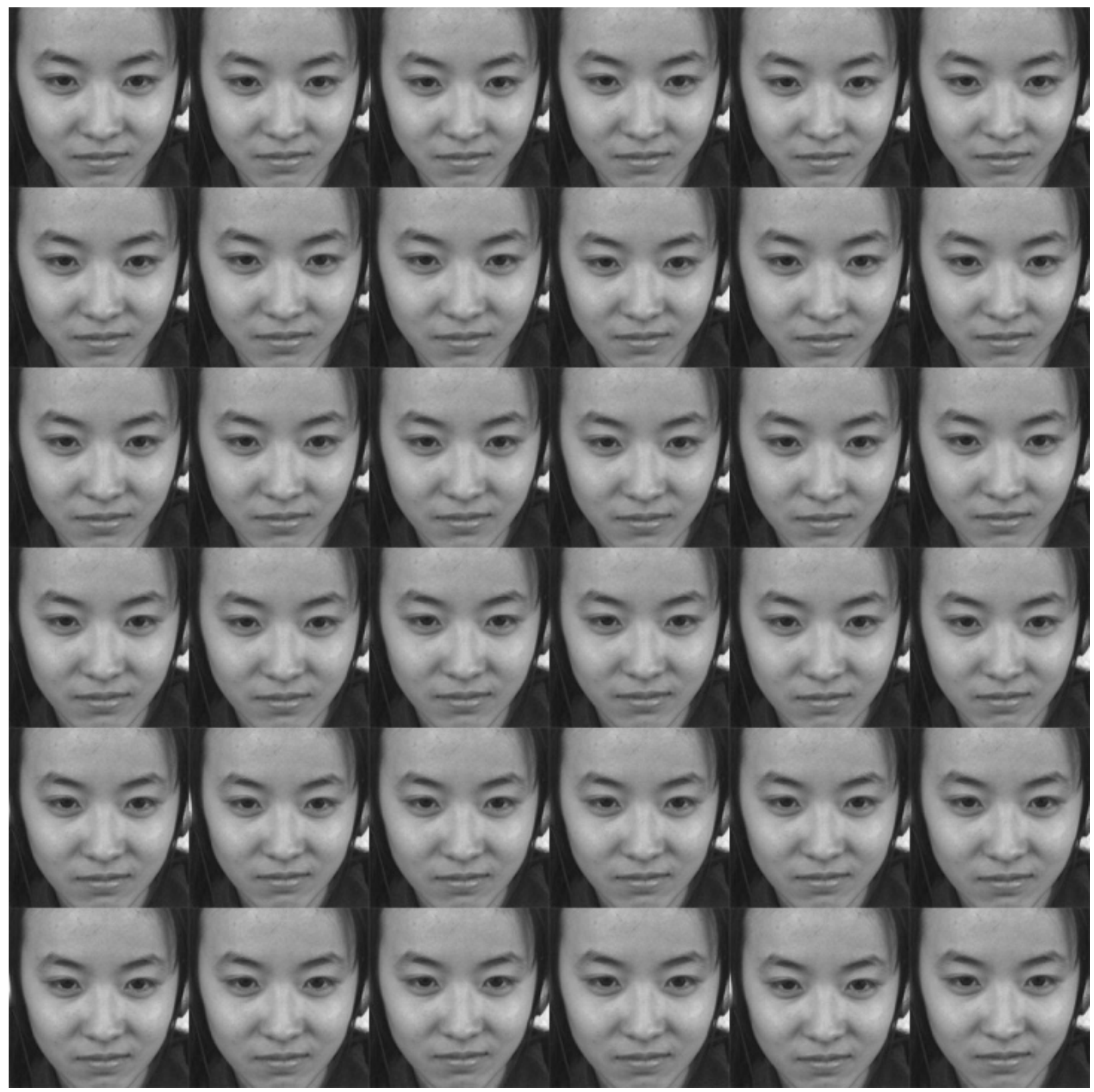

Figure 4. Preprocessed Image Sequence 
CS298 Report

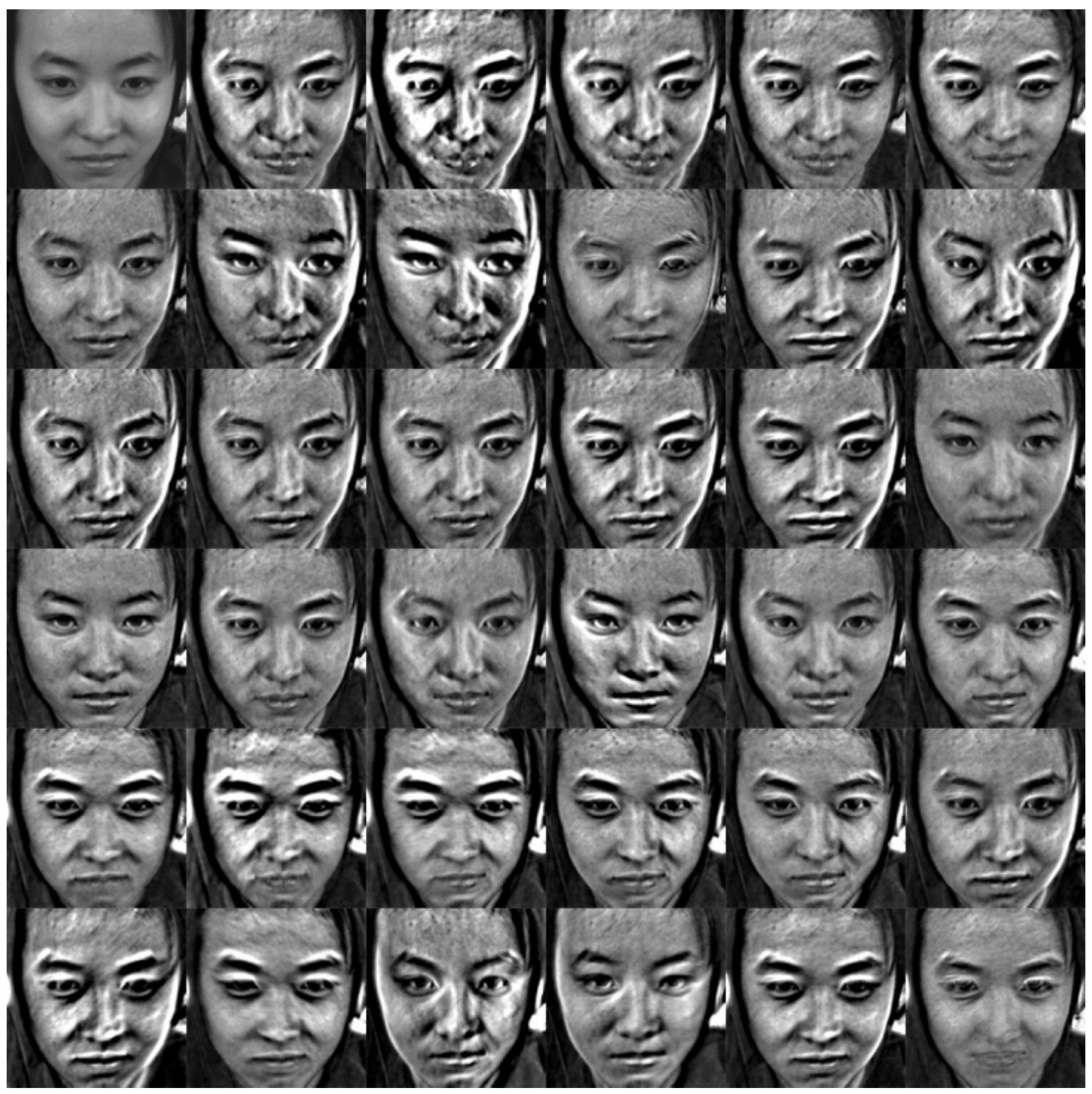

Figure 5. Motion Magnified Image Sequence 


\subsection{Eulerian Video Magnification}

We use the Eulerian Video Magnification (EVM) technique [25], to amplify the subtle emotions that are elicited in the subjects. Following is a brief overview of how the EVM works as described by $\mathrm{Wu}$ et al. in [25]:

1) The input video sequence is first decomposed into various spatial frequency bands

2) A common temporal filter is then applied to the decomposed spatial frequency bands

3) The bands are then magnified by a tunable parameter $\alpha$

4) These magnified bands are then added to the bands obtained after step 2 and are then collapsed to generate the final motion magnified video.

The temporal filters used: Ideal, Butterworth and Second Order Infinite Impulse Response (IIR) and the value of $\alpha$ all affect the overall accuracy of the proposed approach.

$\mathrm{Wu}$ et al. describe the relationship between the temporal processing and motion magnification in detail in [25]. We will briefly describe this relationship here. Let $I(x, t)$ be the intensity of the input image at position $x$ and at time $t$. As the image undergoes translational motion, we can express the intensities as a displacement function $\delta(t)$ such that:

$$
\begin{gathered}
I(x, t)=f(x+\delta(t)) \quad \text { at time } t \\
I(x, 0)=f(x) \quad \text { at time } t=0 .
\end{gathered}
$$

Assuming that the image can be approximated by a first-order Taylor series expansion, we rewrite the image at time $t$ as

$$
I(x, t) \approx f(x)+\delta(t) \frac{\partial f(x)}{\partial x}
$$

Let $B(x, t)$ be the result of applying a temporal filter to the input signal at every position of $x$ in Eq. (1) except at time $t=0$ i.e. at $f(x)$. Thus we have: 


$$
B(x, t)=\delta(t) \frac{\partial f(x)}{\partial x}
$$

As specified above, we amplify the band-passed signal by a magnification factor $\alpha$ and then add it to the original input signal.

$$
\tilde{I}(x, t)=I(x, t)+\alpha B(x, t)
$$

Combining Equations (1), (2) and (3), we get,

$$
\begin{aligned}
\tilde{I}(x, t) & \approx f(x)+(1+\alpha) \delta(t) \frac{\partial f(x)}{\partial x} \\
& \approx f(x)+(1+\alpha) \delta(t)
\end{aligned}
$$

Thus we can see that the displacement in an image sequence $\delta(t)$ is magnified by a factor $(1+\alpha)$ The paper [25] lists three main types of temporal bandpass filters namely: Ideal, IIR \& Butterworth filter. It also provides a general guideline of their typical use cases such as, a filter with a broad passband should be used for motion magnification; while a filter with a narrow passband should be used for color amplification and so on. Figure 5 shows the effect of EVM on preprocessed images. The white highlighted parts in the image are hotspots for magnified motion as compared to the previous image in the sequence. 


\subsection{Spatiotemporal Texture Map based Feature Extraction}

For feature extraction and representation, we use the algorithm proposed by Kamarol et al. [18]. It uses a three dimensional Harris function to extract spatiotemporal information from an image sequence. A block based method is then employed to represent the feature space as a histogram. We shall take a closer look at both these aspects in the following sections.

\subsubsection{Feature Extraction}

The feature extraction employed by Kamarol et al. [18] is as follows:

1) Crop the input image sequence

2) Compute linear scale representation $(L)$ of image sequence using a Gaussian Kernel

3) Compute the convolution of a Gaussian weighting function with a second moment matrix composed of the temporal and spatial derivatives of the first-order derivatives $(\mu)$

4) Calculate the trace and determinant of $\mu$

5) Derive the STTM from $\operatorname{trace}(\mu)$ and $\operatorname{det}(\mu)$

Figure. 6 consolidates the entire approach in a flow diagram: 


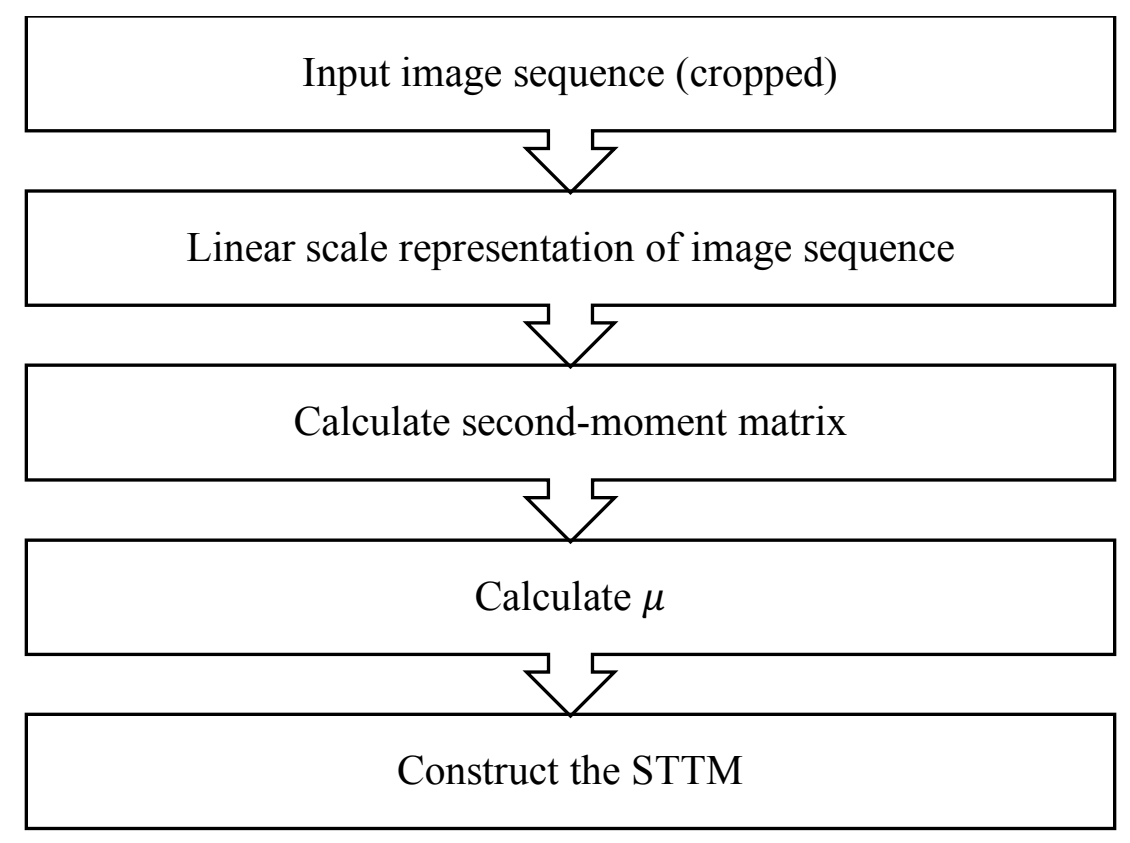

Figure 6. STTM Feature Extraction Process Flow

Let us take a look at each of these steps in more depth.

The given image sequence $f$ is converted into its linear scale-space representation by convoluting it with a three dimensional Gaussian function.

$$
\mathrm{L}\left(\cdot ; \sigma_{1}^{2}, \tau_{1}^{2}\right)=\mathrm{g}\left(\cdot ; \sigma_{1}^{2}, \tau_{1}^{2}\right) * f(\cdot)
$$

$\sigma_{l}^{2}$ where is spatial variance of Gaussian kernel $g$ and $\tau_{l}^{2}$ is the temporal variance.

The spatial features of the input image sequence are represented by $x$ and $y$ which correspond to the respective axes of each image in the sequence while $t$ indicates the temporal information on the time axis. The Gaussian kernel is defined as follows on these three axes.

$$
g\left(x, y, t ; \sigma_{l}^{2}, \tau_{l}^{2}\right)=\frac{\exp \left(-\left(x^{2}+y^{2}\right) / 2 \sigma_{l}^{2}-t^{2} / 2 \tau_{l}^{2}\right)}{\sqrt{(2 \pi)^{3} \sigma_{l}^{4} \tau_{l}^{2}}}
$$


To determine the changes in the spatiotemporal domain in the input image sequence $f$, we move a Gaussian window in various directions by small amounts [4] [24]. To determine the points at which these changes are evident, we apply convolution of a Gaussian weighting function $g\left(\cdot ; \sigma_{l}^{2}, \tau_{l}^{2}\right)$ to a second-moment $3 \times 3$ matrix comprised of first order spatial and temporal derivatives.

$$
\mu=g\left(\cdot ; \sigma_{l}^{2}, \tau_{l}^{2}\right) *\left(\begin{array}{ccc}
L_{x}^{2} & L_{x} L_{y} & L_{x} L_{t} \\
L_{x} L_{y} & L_{y}^{2} & L_{y} L_{t} \\
L_{x} L_{t} & L_{y} L_{t} & L_{t}^{2}
\end{array}\right)
$$

where $L_{x}, L_{y}$ and $L_{t}$ are first order derivatives defined as

$$
\begin{aligned}
& L_{x}\left(\cdot ; \sigma_{i}^{2}, \tau_{i}^{2}\right)=\partial_{x}(g * f), \\
& L_{y}\left(\cdot ; \sigma_{i}^{2}, \tau_{i}^{2}\right)=\partial_{y}(g * f), \\
& L_{t}\left(\cdot ; \sigma_{i}^{2}, \tau_{i}^{2}\right)=\partial_{t}(g * f)
\end{aligned}
$$

where, $\sigma_{i}^{2}=s \sigma_{l}^{2}$ and $\tau_{i}^{2}=s \tau_{l}^{2}$, and $s$ is a constant

Existence of large eigenvalues if $\mu$ indicate the presence of variations in the spatiotemporal domain of the given image sequence. To construct the Harris function $(H)$ using these eigenvalues $\left(\lambda_{1}, \lambda_{2}\right.$ and $\lambda_{3}$ ) we compute the determinant and the trace of $\mu$ as follows:

$$
\begin{aligned}
H & =\operatorname{det}(\mu)-k \operatorname{trace}^{3}(\mu) \\
& =\lambda_{1} \lambda_{2} \lambda_{3}-k\left(\lambda_{1}+\lambda_{2}+\lambda_{3}\right)^{3}
\end{aligned}
$$




\subsubsection{Block-based Feature Representation}

Now that we have extracted the features, we need a method to represent them such that the locality of the variation in the facial expressions in the image sequence is maintained. To do this we first divide each STTM into multiple blocks. Histograms are then calculated for each block which are then concatenated. This forms the feature vector of each of the image sequence.

However, Kamarol et al. [18] found that the feature space is sparse and a histogram computed using uniform width isn't very effective at representing the features of the image sequence. Hence they opted to use an approach with non-uniform bin width, where the data in the lower range is allocated more bins. They achieved this by using the A-law compression before computing the histograms. They claim that this approach has allowed them to capture subtler variations in the image sequence.

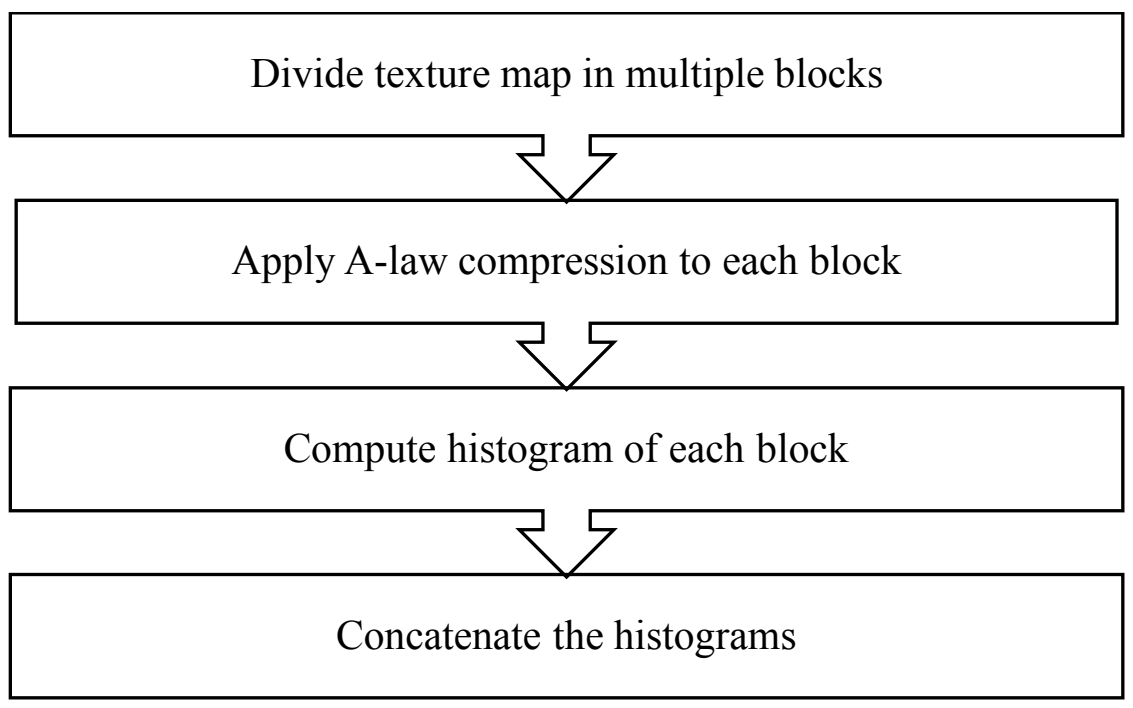

Figure 7. Process flow for block-based feature representation 


\subsection{Classification}

Analyzing the dataset, and the feature space generated by the STTM feature extraction approach, we realize that the number of features are far more than the number of unique, labelled data points. Hence, we came to the conclusion that Support Vector Machines (SVM) is the most logical approach for classification. The CASMEII dataset [29] has five major classes for the identified micro-expressions. Hence we use the multi-class variant SVMs. We also use the oneagainst-one approach instead of the one-against-all approach. In the one-against-one approach, we train $k *(k-1) / 2$ binary classifiers, where $k$ is the number of classes. We then employ a voting scheme while predicting an unseen sample. We employ multiple kernel shapes to find a hyperplane which provides the maximum separation amongst the classes. 


\section{Experiments and Results}

\subsection{Dataset}

As mentioned in Section 3, a variety of datasets exist for macro-expressions exist such as JAFFE [11], CK [7], MMI [16] etc. CK's incremental update, CK+ [10] tries to make up for this by including image sequences that have micro-expressions. However, these image sequences are posed and hence are very harsh and lose the subtlety that is associated with micro-expressions. The CASMEII dataset [29] created by Yan et al. is currently the only spontaneous dataset that is available for micro-expression analysis.

To elicit micro-expressions in the subjects, Yan et al. played some videos that had been carefully preselected and proven to evoke emotions such as beating a pregnant woman, tooth extraction, jokes on stool etc. However, the subjects were instructed to maintain as neutral faces as possible. Failure to do so would incur penalties for subjects. This illusion of high stakes simulated by Yan et al. and the stimulus provided by the videos, elicited micro-expressions in the subjects. The videos were shot using a 200 FPS camera, to capture the rapid and subtle characteristics of micro-expressions. They also used high intensity and constant illumination apparatus to avoid flickering in illumination conditions that is caused due to inconsistent lighting in high FPS video capture.

As Wang et al. [24] have used 247 videos with five expression categories such as Happiness, Surprise, Disgust, Repression and Others (includes emotions such as anger, sadness and tense etc.) we too use these five expression categories to compare our results with the baseline. We leave out videos labelled as Fear. However, there are only two videos labelled as Fear in the CASMEII dataset. 


\subsection{Parameter Selection}

To find out which parameters, work the best for our proposed approach, we tested our hypotheses on smaller prototypes. This provided us with a guideline to work against, while saving the time involved in testing the entire dataset for each parameter and hypothesis. We created an equally supported sample dataset, which had 7 image sequences for each of the 5 expression categories. We conducted our experiments on this dataset, and then used the empirically derived parameters on the entire dataset of 247 videos. As our approach has four major components, we shall discuss the experiments conducted for each of the components in the following sections.

\subsubsection{Preprocessing}

To remove unnecessary regions of the image sequence such as hair, clothing etc. we first crop the image sequences. We first detect the subjects' face in the image sequences using the Viola-Jones face detection algorithm [22]. We then crop the facial area from the image and resized it to $275 \times 275$ pixel size. We then, convert the cropped image to gray-scale. Conversion to grayscale reduces the computational complexity needed during feature extraction. To identify if the cropping and resizing parameter selection affects the accuracy, we cropped the sample dataset to three resolutions $250 \times 250,275 \times 275$ and $300 \times 300$. We then proceeded with the feature extraction, representation and classification using the parameters given by Kamarol et al [18]. We achieved the highest accuracy of $53.2 \%$ for a resolution of $275 \times 275$. Note that we hadn't applied EVM yet. The following Figure. 8 presents the effect of resolution size on accuracy and other parameters haven't been empirically derived to their optimal value. 


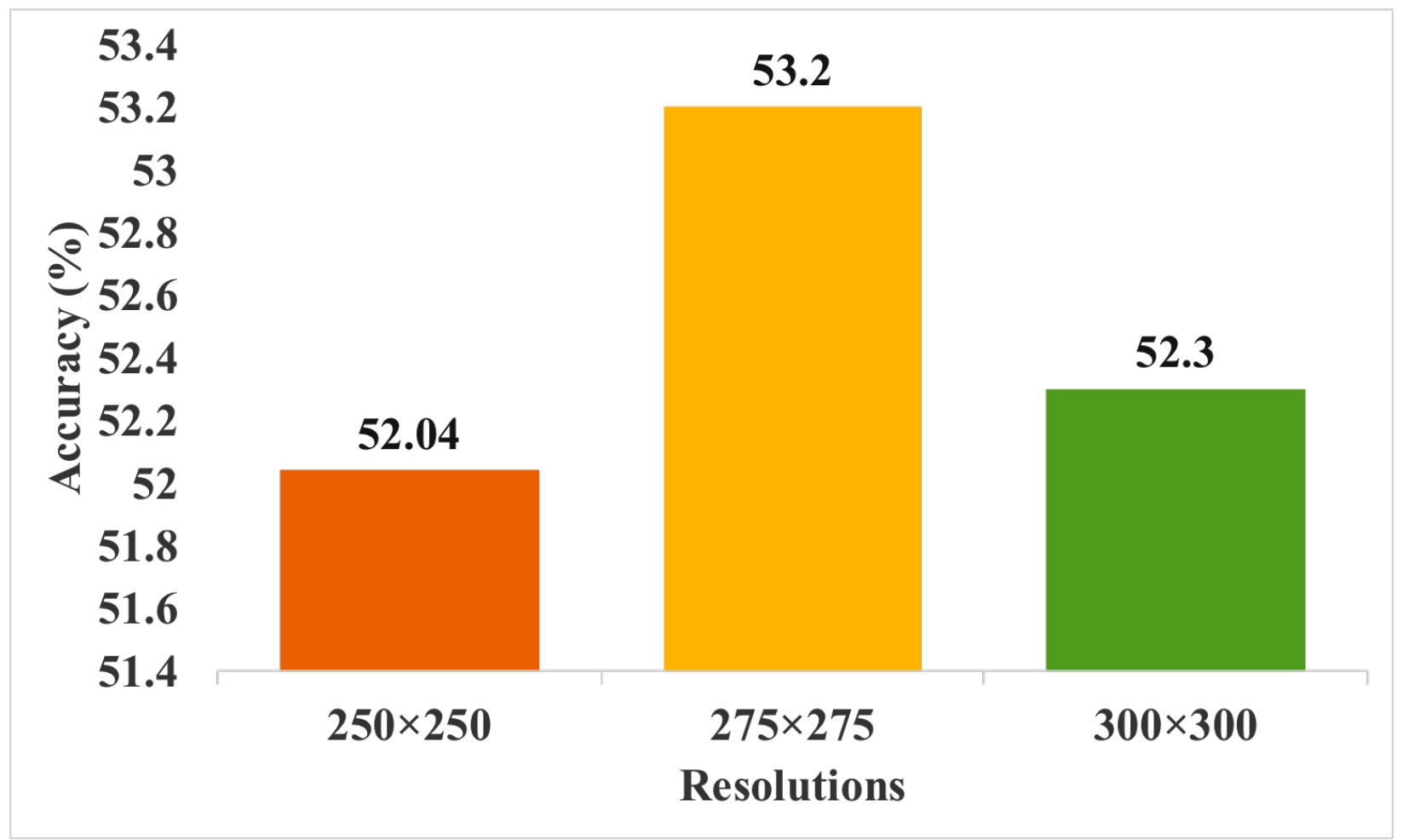

Figure 8. Optimal value for cropping size 


\subsubsection{Eulerian Video Magnification}

There are two parameters that are of prime importance when considering EVM, namely; type of filter and amount of magnification $(\alpha)$. Wang et al. [24] achieved their highest accuracy using a second order IIR filter with $\alpha=20$. To derive these parameters that work best for our approach, we first cropped and resized the images using the parameters derived in Section 4.2.1. We then magnified the motion in these image sequences using EVM. We varied $\alpha$ from 8 to 20 with a step size of two for IIR and Butterworth filters. We then extracted features using STTM parameters described in [18] and then applied Linear SVM for classifying them.

We observed that $\alpha=10$ gives us the best accuracy of $71.43 \%$ for Butterworth filter, viz. in contrast to the findings of Wang et al. [24], where they found that IIR performs better than Butterworth filter at $\alpha=10$. Furthermore, Wang et al. also note that they have achieved the highest accuracy for $\alpha=20$ for the IIR filter. Figure. 9 describes the results of this experiment.

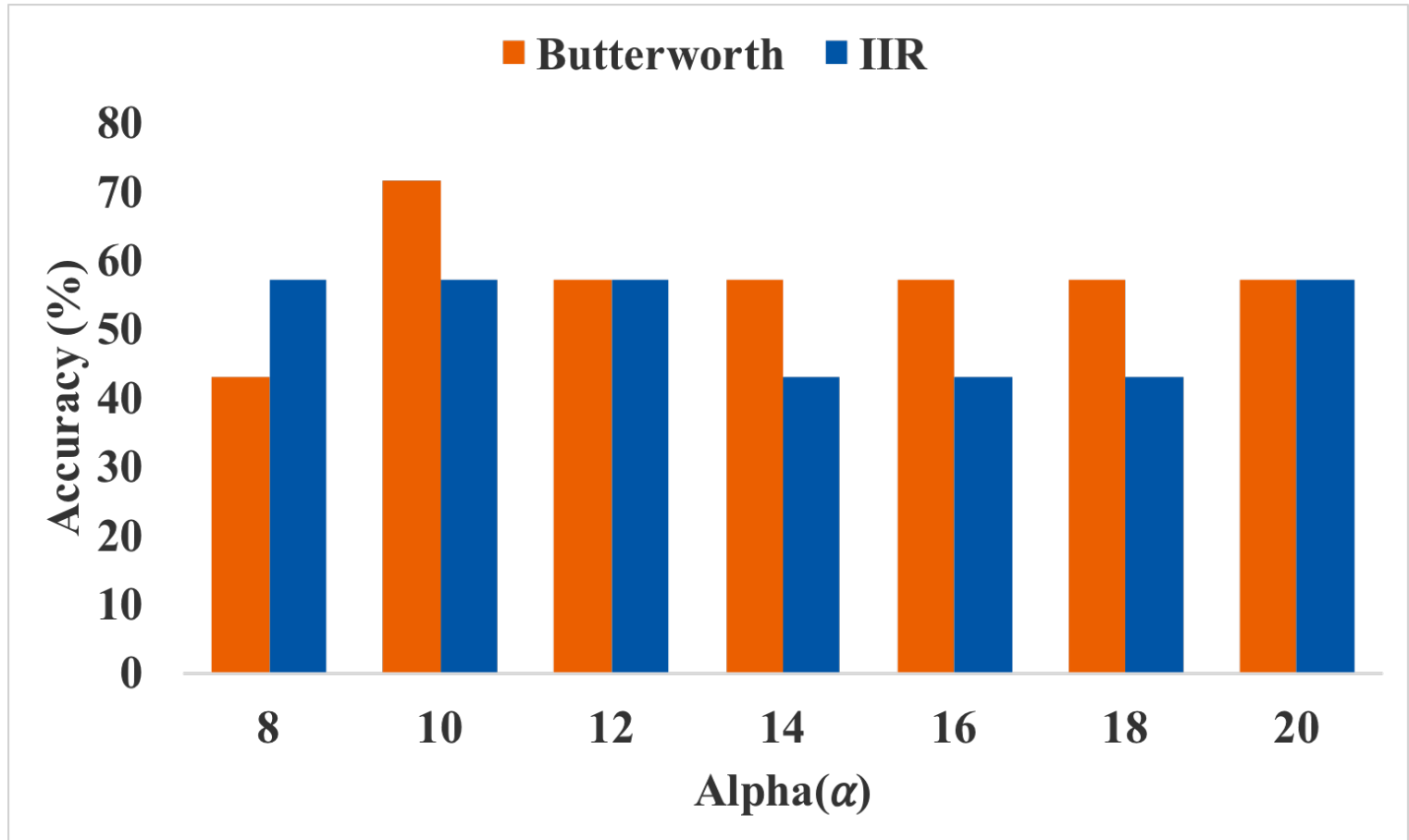

Figure 9. Optimal value for type of filter and $\alpha$ 


\subsubsection{STTM feature extraction and representation}

For feature extraction and representation, we use the optimal parameters achieved by Kamarol et al. [18] for CASMEII dataset.

They are as follows:

$$
\begin{aligned}
& \sigma_{1}^{2}=2 \\
& \tau_{1}^{2}=2 \\
& k=0.04 \\
& A=28 \\
& \text { number of bins }=10 \\
& \text { number of blocks }=9 \times 9 \\
& \text { overlapping ratio }=30 \%
\end{aligned}
$$




\subsubsection{Classification}

Wang et al. have used leave-one-out-cross (LOOCV) for validating their method. They have also utilized various kernel shapes such a Linear, RBF and Polynomial for their SVMs. However, this method is very time consuming as it trains the model on $n-1$ samples and then tests it on the remaining sample. This procedure is repeated for all the $n$ samples in the dataset. Instead we opted for K-Fold cross validation which is much quicker compared to LOOCV. We preprocess, extracted and represented features using the parameters we derived so far. Then, we applied SVM classification using one-against-one approach on various kernel shapes and varying number of splits for K-Fold validation. We achieved the highest accuracy of $71.4 \%$ for linear and RBF kernels using 10-fold validation. Figure. 10 illustrates our findings.

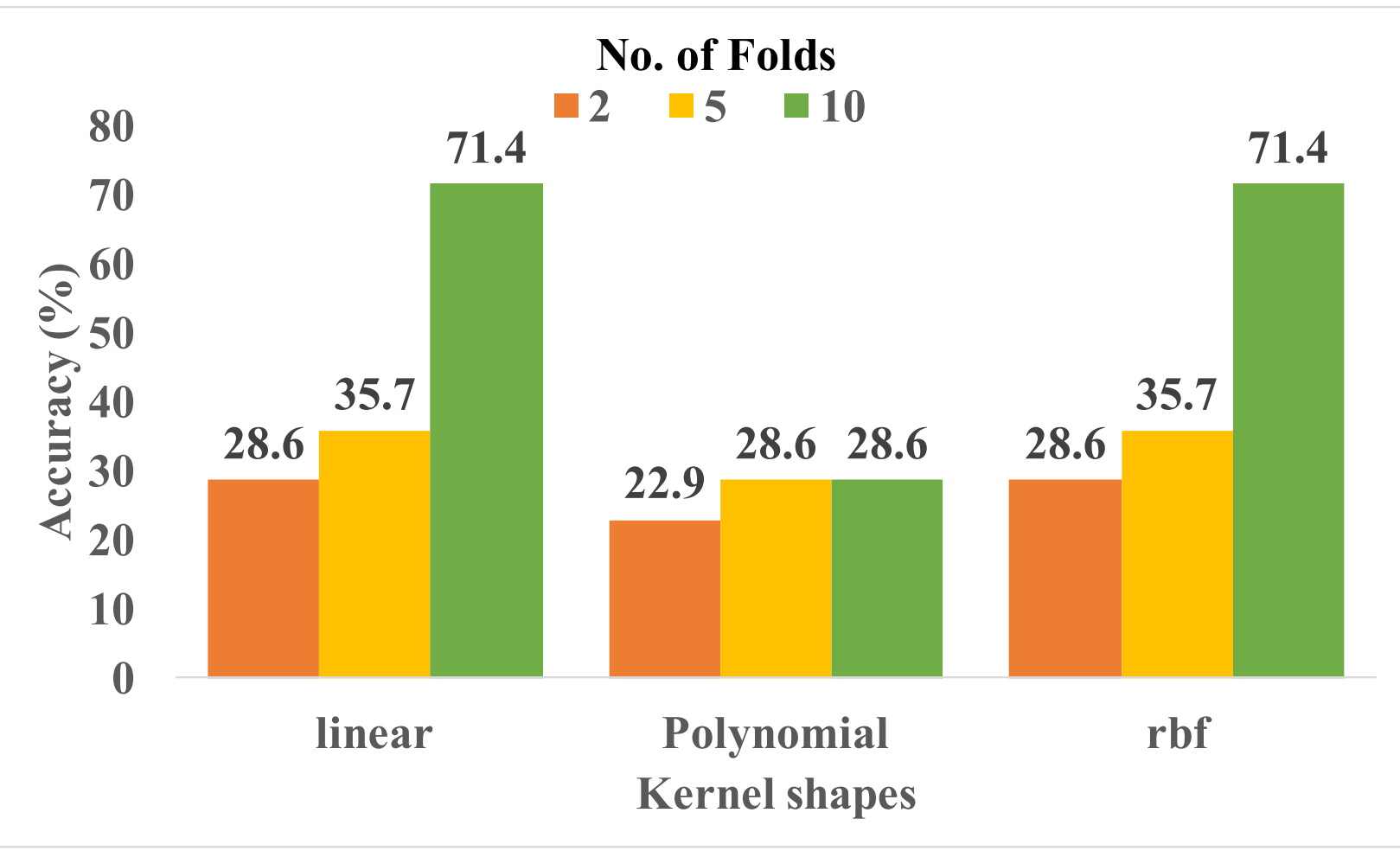

Figure 10. Optimal values of Kernel Shape and K-Fold validation 


\subsection{Final results on complete pipeline}

Now that we have derived all the parameters' optimal value, we apply these parameters in the respective phases of the pipeline described in Figure. 1 on the entire CASMEII dataset [29] of 247 videos. We repeat the classification 10000 times and take the average. We achieve an accuracy of $80 \%$ with linear and RBF kernels viz. about $5 \%$ higher than the baseline. The results validate that the intermediate results achieved on prototypes were a step in the right direction. Maximum accuracy achieved is $80 \%$.

Table 1. Classification Report for 5 Emotion Categories

\begin{tabular}{|c|c|c|c|c|}
\hline & Precision & Recall & F1-score & Support \\
\hline Disgust & 0.0 & 0.0 & 0.0 & 1 \\
\hline Happiness & 0.0 & 0.0 & 0.0 & 20 \\
\hline Others & 0.8 & 1.0 & 0.89 & 1 \\
\hline Repression & 0.0 & 0.0 & 0.0 & 1 \\
\hline Surprise & 0.0 & 0.0 & 0.0 & $\mathbf{2 5}$ \\
\hline Avg./Total & $\mathbf{0 . 6 4}$ & $\mathbf{0 . 8}$ & $\mathbf{0 . 7 1}$ & \\
\hline
\end{tabular}




\section{Conclusion and Future Work}

In this paper, we proposed a new approach to motion magnification and feature extraction. While Wang et al. [24] had used LBP-TOP feature extraction, we extracted dynamic textural information using Spatiotemporal Texture Map (STTM) to identify micro-expressions. We achieved an accuracy of $80 \%$ using linear and RBF kernels viz. is about a 5\% increase compared to the baseline [24].

Future forays in this area would be to tune the parameters used in STTM feature extraction and representation and find out if they improve the result. Multi-tier SVM classification could also be applied to combat the problem of unequal support of micro-expression samples of each category. We could also apply deep neural network for classification. 


\section{References}

[1] A. Dhall, "Collecting large, richly annotated facial-expression databases from movies," 2012.

[2] P. Ekman, "Darwin, deception, and facial expression," in Anonymous 2003, . DOI: 10.1196/annals.1280.010.

[3] P. Ekman, M. O'Sullivan and M. G. Frank, "A Few Can Catch a Liar," Psychol. Sci., vol. 10, (3), pp. 263-266, 1999. Available: https://doi.org/10.1111/1467-9280.00147. DOI: $10.1111 / 1467-9280.00147$.

[4] W. Förstner and E. Gülch, "A fast operator for detection and precise location of distinct points, corners and centres of circular features," in Proc. ISPRS Intercommission Conference on Fast Processing of Photogrammetric Data, 1987,.

[5] M. Frank et al, "I see how you feel: Training laypeople and professionals to recognize fleeting emotions," in The Annual Meeting of the International Communication Association. Sheraton New York, New York City, 2009, .

[6] C. Harris and M. Stephens, "A combined corner and edge detector." in Alvey Vision Conference, 1988, .

[7] T. Kanade, J. F. Cohn and Y. Tian, "Comprehensive database for facial expression analysis," in Automatic Face and Gesture Recognition, 2000. Proceedings. Fourth IEEE International Conference on, 2000, .

[8] W. Kienzle et al, Face Detection - Efficient and Rank Deficient. 200517.

[9] C. Liu, Beyond Pixels: Exploring New Representations and Applications for Motion Analysis, 2009. 
[10] P. Lucey et al, "The extended cohn-kanade dataset (ck ): A complete dataset for action unit and emotion-specified expression," in Computer Vision and Pattern Recognition Workshops (CVPRW), 2010 IEEE Computer Society Conference on, 2010, .

[11] M. J. Lyons et al, "The japanese female facial expression (JAFFE) database," in Proceedings of Third International Conference on Automatic Face and Gesture Recognition, 1998, .

[12] M. Lyons et al, "Coding facial expressions with gabor wavelets," in Proceedings Third IEEE International Conference on Automatic Face and Gesture Recognition, 1998, . DOI: 10.1109/AFGR.1998.670949.

[13] D. Matsumoto and H. S. Hwang, "Evidence for training the ability to read microexpressions of emotion," Motiv. Emotion, vol. 35, (2), pp. 181-191, 2011. Available: https://doi.org/10.1007/s11031-011-9212-2. DOI: 10.1007/s11031-011-9212-2.

[14] A. Mehrabian, "Communication without words," Communication Theory, pp. 193-200, 2008.

[15] T. Ojala, M. Pietikainen and T. Maenpaa, "Multiresolution gray-scale and rotation invariant texture classification with local binary patterns," IEEE Trans. Pattern Anal. Mach. Intell., vol. 24, (7), pp. 971-987, 2002.

[16] M. Pantic et al, "Web-based database for facial expression analysis," in Multimedia and Expo, 2005. ICME 2005. IEEE International Conference on, 2005, .

[17] T. A. Russell, E. Chu and M. L. Phillips, "A pilot study to investigate the effectiveness of emotion recognition remediation in schizophrenia using the micro-expression training tool," British Journal of Clinical Psychology, vol. 45, (4), pp. 579-583, 2006. 
[18] S. K. A. Kamarol et al, "Spatiotemporal feature extraction for facial expression recognition," IET Image Processing, vol. 10, (7), pp. 534-541, 2016. . DOI: 10.1049/ietipr.2015.0519.

[19] G. Sandbach et al, "Static and dynamic 3D facial expression recognition: A comprehensive survey," Image Vision Comput., vol. 30, (10), pp. 683-697, 2012. Available: $\quad$ http://www.sciencedirect.com/science/article/pii/S0262885612000935. DOI: https://doi.org/10.1016/j.imavis.2012.06.005.

[20] E. Schopler, G. B. Mesibov and L. J. Kunce, Asperger Syndrome Or High-Functioning Autism? 1998.

[21] C. Tomasi and T. Kanade, "Detection and tracking of point features," 1991.

[22] P. Viola and M. J. Jones, "Robust real-time face detection," International Journal of Computer Vision, vol. 57, (2), pp. 137-154, 2004.

[23] Y. Wang et al, "Efficient spatio-temporal local binary patterns for spontaneous facial microexpression recognition," PloS One, vol. 10, (5), pp. e0124674, 2015.

[24] Y. Wang et al, "Effective Recognition of Facial Micro-expressions with Video Motion Magnification," Multimedia Tools Appl, vol. 76, (20), pp. 21665-21690, 2017. Available: https://doi.org/10.1007/s11042-016-4079-6. DOI: 10.1007/s11042-016-4079-6.

[25] H. Wu et al, "Eulerian Video Magnification for Revealing Subtle Changes in the World," ACM Trans.Graph., vol. 31, (4), pp. 65:1-65:8, 2012. Available: http://doi.acm.org/10.1145/2185520.2185561. DOI: $10.1145 / 2185520.2185561$.

[26] Q. Wu, X. Shen and X. Fu, "The machine knows what you are hiding: An automatic microexpression recognition system," in Proceedings of the 4th International Conference on 
Affective Computing and Intelligent Interaction - Volume Part II, 2011, Available: http://dl.acm.org/citation.cfm?id=2062850.2062867.

[27] X. Li et al, "A spontaneous micro-expression database: Inducement, collection and baseline," in 2013 10th IEEE International Conference and Workshops on Automatic Face and Gesture Recognition (FG), 2013, . DOI: 10.1109/FG.2013.6553717.

[28] X. Li et al, "Towards Reading Hidden Emotions: A Comparative Study of Spontaneous Micro-expression Spotting and Recognition Methods," IEEE Transactions on Affective Computing, vol. PP, (99), pp. 1-1, 2017. . DOI: 10.1109/TAFFC.2017.2667642.

[29] W. Yan et al, "CASME II: An improved spontaneous micro-expression database and the baseline evaluation," PloS One, vol. 9, (1), pp. e86041, 2014.

[30] W. Yan et al, "How Fast are the Leaked Facial Expressions: The Duration of MicroExpressions," J. Nonverbal Behav., vol. 37, (4), pp. 217-230, 2013. Available: https://doi.org/10.1007/s10919-013-0159-8. DOI: 10.1007/s10919-013-0159-8.

[31] G. Zhao and M. Pietikainen, "Dynamic texture recognition using local binary patterns with an application to facial expressions," IEEE Trans. Pattern Anal. Mach. Intell., vol. 29, (6), pp. 915-928, 2007.

[32] D. Matsumoto, L. G. Skinner and H. Hwang, "Reading People: Behavioral Anomalies and Investigative Interviewing," The FBI Law Enforcement Bulletin, 2015.

[33] Handeyside, H. (2015). Be careful with your face at airports (Opinion) - CNN. [online] CNN. Available at: https://www.cnn.com/2015/03/19/opinions/handeyside-tsa-spotprogram/index.html [Accessed 5 May 2018]. 